Urologe 2010 · 49:907-909

DOI 10.1007/s00120-010-2300-1

Online publiziert: 24. Juli 2010

(c) Springer-Verlag 2010

\author{
J.A. Steffens \\ Zertifiziertes Prostatakarzinomzentrum, Klinik für Urologie und Kinderurologie, \\ St. Antonius-Hospital Eschweiler, \\ Akademisches Lehrkrankenhaus der RWTH Aachen, Eschweiler
}

\title{
Auf Spurensuche
}

in einem zertifizierten Prostatakarzinomzentrum besteht, unbeantwortet bleiben.

In den politischen Sonntagsreden wird das Qualitätsprinzip als Leitbild des Gesundheitswesens anerkannt. Im Alltag aber hat es viel von seinem Glanz verloren.

\section{- In Deutschland fehlt ein klares Bekenntnis zum Wettbewerbsprinzip.}

Qualitätssicherung wird zwar im Sozialgesetzbuch von allen Ärzten verpflichtend gefordert, es gibt jedoch in unserem Gesundheitssystem keine Anreize zur Qualitätssteigerung und Kostensenkung. Die Gründung zertifizierter Prostatakarzinomzentren folgte initial Marketingaspekten. In Anbetracht jährlicher Zentrumskosten von 150.000 EUR/Jahr kam es jedoch rasch zur ernüchternden Feststellung, dass sich durch Etablierung eines Zentrums weder die Patientenzahlen noch die Erlösgewinne steigern ließen. Deshalb bedarf es einer Neuregelung durch die Politik und eines klaren Bekenntnisses zum Wettbewerbsprinzip. Nur in den Einrichtungen, in denen die Kernforderungen der Erhebungsbögen für Prostatakarzinomzentren erfüllt werden, dürfen künftig Zertifikate erteilt und Rezertifizierungen ausgesprochen werden. Wenn keine interdisziplinäre und sektorübergreifende Kooperation nachweisbar ist und Nachsorgedaten nicht kontinuierlich erhoben werden, fehlen die Genehmigungskriterien für ein Zentrum. Die Aufsichtsfunktion von OnkoZert muss sich künftig stärker an der Erfüllung der wesentlichen Qualitätsforderungen orientieren.
Das Prostatakarzinom ist die einzige Tumorentität, für die alternativ zum Monopolisten OnkoZert von Urologen ein zweites Zertifizierungsverfahren etabliert wurde. Der Dachverband der Prostatazentren (DVPZ) verfolgt die gleichen Ziele, erfreut sich großer Akzeptanz und zertifiziert im Gegensatz zu den Karzinomzentren der deutschen Krebsgesellschaft auch die gutartigen und entzündlichen Erkrankungen der Prostata. Die Analyse von Zumbé und Berges lässt vergleichbare Defizite wie bei den DKG-zertifizierten Prostatakarzinomzentren erkennen. Dem DVPZ erschließt sich zwar die gleiche Zielgruppe wie für die DKGzertifizierten Zentren, jedoch ist ihr Zertifikat im nationalen Krebsplan nicht berücksichtigt. Diese fehlende Anerkennung könnte somit die Planungssicherheit gefährden und zu einer kleinen tektonischen Verschiebung führen: Kliniken werden sich künftig nur einem Zertifizierungsverfahren unterziehen, welches staatlich anerkannt ist. Derzeit verknüpfen sich Teilmärkte, die bisher nicht verbunden waren. Die Protagonisten beider Zertifizierungseinrichtungen suchen die Zusammenarbeit und rücken zusammen.

Eine Folge der hochtechnisierten Medizintechnik ist die Marktreife des Operationsroboters Da Vinci.

\section{- Die Etablierung von ca. 50 Groß- geräten hat zu einer Polarisierung in der deutschen Urologie geführt.}

Eine ähnliche Entwicklung gab es vor 25 Jahren mit der Einführung der ESWL. Kliniken, die sich die Anschaffung des inbar werden, wird die Gretchenfrage, ob ein Behandlungs- und Überlebensvorteil 
itial defizitären und im DRG-System bisher nicht abgebildeten Großgerätes nicht leisten können, scheinen Wettbewerbsnachteile zu haben. Die Angst vieler urologischer Klinikchefs, aufgrund mangelnder Attraktivität weniger radikale Prostatektomien durchführen zu können, ist begründet. Gegenwärtig werden in Kliniken ohne ein Da Vinci-System 30\% weniger radikale Prostatektomien durchgeführt. Obwohl die offene retropubische radikale Prostatektomie weiterhin Goldstandard ist und sich die neue Technik erst im Langzeitverlauf behaupten muss, ist der Da Vinci ein Magnet mit Sogwirkung auf die Patienten. Leider definieren wir Urologen uns seit Jahren nur über die Zahl der radikalen Prostatektomien pro Jahr. Wer <10o Patienten jährlich operiert, wird oft belächelt.

Die gegenwärtige Entwicklung hat uns Operateure wachgerüttelt. Denn unser faszinierendes Fach bietet so viel mehr! Operationstechnische Herausforderungen sind die im DRG-System gut gepreisten ablativen und rekonstruktiven Verfahren unter Inkorporation von Darmsegmenten und Mundschleimhaut. Die endourologischen, transurethralen und perkutanen Verfahren sind zwar niedrig gepreist, stellen jedoch das „Brot- und Buttergeschäft“ vieler urologischer Kliniken dar und müssen täglich geübt und gepflegt werden, um eine gute Ergebnisqualität zu erzielen. Im Beitrag von Herrn Hakenberg wird der Stellenwert des OP-Roboters kritisch dargestellt.

Das Thema „Ärztliche Weiterbildung“ ist von höchstem Interesse für unsere jungen Mitarbeiter und von größter Bedeutung für die Strukturqualität der Medizin in Deutschland. Für die Ärztekammern ist die Analyse der Weiterbildungsinhalte gegenwärtig das Topthema.

- In Anbetracht des Mangels an qualifiziertem ärztlichem Nachwuchs können Kliniken und Praxen ihre Attraktivität durch Weiterbildungsermächtigungen steigern.

Herr Gschwend stellt die ernüchternde Realität in der deutschen Uroonkologie dar. Die zunehmende interdisziplinäre Zentralisierung der Versorgung onkologischer Patienten und das Inkrafttreten der neuen Onkologievereinbarung im Januar 2010 erfordern einen hohen Grad der Spezialisierung, der bisher von Urologen nicht ausreichend genutzt wurde. Trotz eines umfangreichen Angebots an Fortbildungsmöglichkeiten existieren $\mathrm{zu}$ wenige Weiterbildungsstätten für die „Medikamentöse Tumortherapie“. Wenn von Urologen jedoch keine flächendeckende Weiterbildung in dieser Subspezialität angeboten werden kann, wird dieses Teilgebiet für die deutsche Urologie (ähnlich wie in den USA) verloren gehen.

Eine weitere Initiative haben die Landesärztekammern bundesweit mit der Bundesärztekammer 2009 gestartet. Erstmals gab es eine Evaluation der Weiterbildung in ganz Deutschland. Fast 30.000 Ärzte beantworteten bei einer Online-Umfrage Fragen zur Weiterbildung. Das Ergebnis war zufriedenstellend. Allerdings wurde auch deutlich, dass hohe Arbeitsbelastung, Bürokratie und Überstunden den Berufsalltag der Ärzte prägen. In den Beiträgen von Gschwend und Kliesch wird deutlich, dass bei Eintritt der Weiterbildung lediglich die Hälfte aller Weiterzubildenden vom Weiterbildungsbefugten einen strukturierten Weiterbildungsplan erhält. Nahezu die Hälfte der Weiterzubildenden gab an, dass keine Lern- bzw. Weiterbildungsziele vereinbart wurden. Beklagt wurden zudem mangelnde Betreuung während der Weiterbildung und zu geringe Möglichkeiten für wissenschaftliche Arbeiten. Diese und andere Defizite werden anhand der Evaluation der Landesärztekammern erfasst und müssen Ansporn zur Verbesserung sein. Denn nur so können wir unserer Verantwortung für eine gute Patientenversorgung der Zukunft und für unseren ärztlichen Nachwuchs gerecht werden. Allerdings können Weiterbildungsinhalte nur dann konsequent und umfassend vermittelt werden, wenn alle Klinikstellen besetzt sind. Denn in Anbetracht der 5000 unbesetzten Arztstellen in deutschen Krankenhäusern bestehen aufgrund der gesetzlichen Forderung nach Umsetzung des Arbeitszeitgesetzes personelle Engpässe, die meist nur die tägliche Erfüllung der Kernaufgaben ermöglichen.

Die Assistentenzufriedenheit wird verständlicherweise nur dort hoch sein, wo alle Stellen besetzt sind, die Weiterbil- dungsinhalte und Zusatzweiterbildungsinhalte erfüllt werden, das Arbeitszeitgesetz umgesetzt ist, familienfreundliche Arbeitsbedingungen bestehen und ein gutes Betriebsklima herrscht. Die Analyse von Herrn Enzmann macht deutlich, dass nur 50\% der Ärzte mit den gegenwärtigen Bedingungen am Arbeitsplatz zufrieden sind und sich nur 40\% mit dem Arbeitgeber identifizieren.

\section{- Nicht das Krankenhaus als Institution, sondern Ruf und Können des Klinik- chefs und der Oberärzte sind Motivationsfaktoren für junge Kolleginnen und Kollegen zum Eintritt in die Abteilung.}

Nur persönliche Vorbildfunktionen, Vorleben eines Arztbildes, welches den Menschen in den Mittelpunkt stellt, Vermittlung einer fundierten operativen Ausbildung und wissenschaftliche Nachwuchsförderung sind Beweggründe für die Anstellung in der Klinik. Die aufgezeigten Defizite sind individuell in jeder Einrichtung zu analysieren. Der konsequente Abbau der Mängelliste wird die Arbeitsplatzsituation verbessern und die Attraktivität der Abteilung steigern.

Medizinische Versorgungszentren (MVZ) stellen ein politisches Instrument zur Bündelung der ärztlichen Versorgung, Heben von Wirtschaftlichkeitsreserven und Beseitigung einer postulierten Über- und Fehlversorgung in der ambulanten Medizin dar. Sie können gerade in fachärztlich unterversorgten Regionen eine sinnvolle Kooperationsform sein. In einigen Gebieten sind sie jedoch ein unerwünschter Fremdkörper, der zweckentfremdet der Renditesteigerung, Gewinnoptimierung, Zuweisungslenkung und Kostenverlagerung dient. Diese Ergebnisse stehen jedoch im Widerspruch zum körperschaftlich verfassten ambulanten Versorgungssystem. Herr Bloch analysiert Ursachen und Folgen der noch unter sozialdemokratischer Regierungsverantwortung initiierten MVZ. Die qualitativ hochwertige ambulante Versorgung kann künftig nur gewährleistet werden, wenn freie Arztwahl der Versicherten, Therapiefreiheit in wirtschaftlicher Verantwortung und Freiberuflichkeit des Arztes gesichert bleiben. Daran orientiert sich er- 
freulicherweise auch die Vereinbarung im Koalitionsvertrag der schwarz-gelben Regierung. In Anbetracht des drohenden Defizits der gesetzlichen Krankenkassenversorgung von $11 \mathrm{Mrd}$. EUR ist jedoch zu erwarten, dass das Problem der mangelnden Einnahmen der GKV mit Sparmaßnahmen auf der Ausgabenseite gelöst werden soll. Wirtschaftlichkeitsreserven werden jedoch nicht durch dirigistische, staatlich verordnete und kooperationsgefährdende Maßnahmen gehoben. In Anbetracht des zunehmenden Mangels an qualifiziertem ärztlichem Nachwuchs sind neue Kooperationsformen zwar wünschenswert, jedoch nur im Einvernehmen mit der Ärzteschaft zu erreichen.

Aus juristischer Sicht stellen Schramm u. Gierthmühen ausgewählte Konstellationen dar, die aktuelle, von der Rechtssprechung bisher ungeklärte Fragestellungen zum MVZ betreffen. Im neuen Koalitionsvertrag wird ungewöhnlich konkret formuliert, dass die Geschäftsanteile nur von Ärzten gehalten werden können. Bisher wurde nur ein MVZ unter ärztlicher Leitung verlangt. Sollte dieser neue Ansatz tatsächlich umgesetzt werden, so dürfte vielerorts ein Wechsel auf Eigentümerseite bevorstehen. Durch die jüngsten Änderungen des Vertragsarztrechtes sind die bisher privilegierten MVZ den Berufsausübungsgemeinschaften wieder weitgehend gleichgestellt worden. Die neue Rolle der MVZ im Gesundheitssystem bleibt abzuwarten.

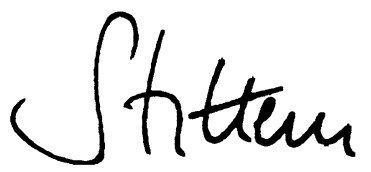

J.A. Steffens

\section{Korrespondenzadresse \\ Prof. Dr. J.A. Steffens}

Zertifiziertes Prostatakarzinomzentrum, Klinik für Urologie und Kinderurologie,

St. Antonius-Hospital Eschweiler,

Akademisches Lehrkrankenhaus der RWTH Aachen,

Dechant-Deckers-Straße 8, 52249 Eschweiler

joachim.steffens@sah-eschweiler.de

\section{Jahreskongress 2010 Männergesundheit}

9. Oktober 2010,

KongressCenter Bad Homburg

Neue Wege beschreitet die Deutsche Gesellschaft für Mann und Gesundheit (DGMG) auf ihrem „Jahreskongress 2010 Männergesundheit". Unter dem Motto "Offener Horizont" ist ein interaktiver Ärzte-Kongress geplant, bei dem auch die Teilnehmer mitdiskutieren. Eingeladen zu diesem Kongress sind alle Ärzte, die sich mit dem Thema Männergesundheit befassen. Das sind Urologen und Andrologen ebenso wie Kardiologen, Sport- und Ernährungsmediziner, Psychologen sowie Internisten und Allgemeinmediziner. Am 9. Oktober 2010 finden im KongressCenter Bad Homburg wissenschaftliche Vorträge, Podiumsdiskussionen und Workshops unter anderem zu den Themen Männersexualität, Prostata-Erkrankungen, Onkologie in der Urologie, Ernährungsmedizin für den Mann sowie Burnout und Depressionen statt.

\section{Wichtige Zertifizierungspunkte}

„Wie hätte ich entschieden?" heißt es in der interaktiven Podiumsdiskussion, in der verschiedene Fallbeispiele gemeinsam mit dem Publikum diskutiert und „,behandelt" werden. Die Ärzte erhalten die Möglichkeit über ein Kommunikationssystem aktiv an Entscheidungsprozessen teilzunehmen. Der Kongress wird mit acht bis zehn Punkten zertifiziert.

\section{Onkologie in der Urologie}

Ein wichtiger Kongress-Schwerpunkt ist die Onkologie in der Urologie, bei dem es unter anderem um Prävention, neue Behandlungsmethoden und Rehabilitation geht. Infektiologie und Aids stehen ebenso auf dem Tagungsprogramm wie die Behandlung des benignen ProstataKarzinoms. Auch das Thema Gebrechlichkeit, das immer mehr zu einem gesellschaftlichen Problem wird, ist Thema des Jahreskongresses 2010. Über die Rolle von Testosteron auf diesem Feld wird ebenfalls diskutiert.
Neben dem medizinischen Bereich ist das Thema Marketing und Praxis-PR ein weiterer Schwerpunkt des Kongresses.

\section{Außergewöhnliche Männer- gesundheitspraxen gesucht} In diesem Jahr geben wir erstmals allen „Männerärzten“ die Möglichkeit sich aktiv am Kongress zu beteiligen. Gesucht werden drei außergewöhnliche Männergesundheitspraxen, die sich durch besondere Aktivitäten für die Männergesundheit und ihren Auftritt nach außen auszeichnen.

Eine Jury prämiert die drei kreativsten und aktivsten Männergesundheitspraxen. Ihre Konzepte werden auf unserem Jahreskongress vorgestellt. Als Preis erhalten die drei ausgezeichneten Praxen ein kostenloses Medientraining „Life vor der Kamera“, bei dem der richtige Auftritt vor Publikum erlernt wird. Außerdem werden die drei besten Praxis-Konzepte in der Ärzte Zeitung, unserem Medien-Kooperationspartner, vorgestellt.

Die zehn besten Praxiskonzepte erhalten für jeweils zwei Personen freien Eintritt zum Jahreskongress 2010 Männergesundheit in Bad Homburg. Unterstützt wird diese Aktion von der Apogepha Arzneimittel $\mathrm{GmbH}$ in Dresden.

Bewerbungsunterlagen können bei der Geschäftsstelle angefordert oder unter www.mann-und-gesundheit.com/Jahreskongress2010/Bewerbungsbogen heruntergeladen werden.

Ärzte können sich ab sofort anmelden bei:

Deutsche Gesellschaft für Mann und

Gesundheit,

Friedberger Str. 2, 61350 Bad Homburg

Tel.: (0 61 72) 9661 31,

mail@mann-und-gesundheit.com

www.mann-und-gesundheit.com 